\title{
Timolol Activates the Enzyme Activities of Human Carbonic Anhydrase I and II
}

\author{
Ayako Sugimoto, ${ }^{a}$ Hiroaki Ikeda, ${ }^{*, a}$ Hidetoshi Tsukamoto, ${ }^{b}$ Kenji Kinira, ${ }^{a}$ Manabu Ishioka, ${ }^{c}$ \\ Junzo Hirose, ${ }^{d}$ Toshiyuki Hata, ${ }^{d}$ Haruto FujIOKA, ${ }^{d}$ and Yukio OnO ${ }^{d}$ \\ ${ }^{a}$ Department of Pharmaceutical Services, Hiroshima University Hospital; 1-2-3 Kasumi, Minami-ku, Hiroshima \\ 734-8551, Japan: ${ }^{b}$ Takayama Eye Clinic; 2-3-15 Shinonome-Honmachi, Minami-ku, Hiroshima 734-0023, Japan: \\ ${ }^{c}$ Department of Applied Biological Science, Fukuyama University; and ' Faculty of Pharmacy and Pharmaceutical \\ Sciences, Fukuyama University; Gakuen-cho, Fukuyama 729-0292, Japan.
}

Received June 15, 2009; accepted November 8, 2009; published online November 16, 2009

Timolol, a beta-blocker, has been shown to be an effective ocular hypotensive agent when used alone or with carbonic anhydrase inhibitor on ocular hypertensive or open angle glaucoma patients. The effect of timolol hemihydrate on the $\mathrm{CO}_{2}$ hydration activities of human carbonic anhydrase (HCA) I and II and their reaction mechanisms were investigated. Timolol activates the enzyme activities of HCA I and HCA II. In HCA I and II, the enzyme kinetic results clearly showed that timolol increases the value of $V_{\max }$ but does not influence the value of $K_{\mathrm{m}}$. The enzyme kinetic method showed that timolol noncompetitively activates HCA I and II activities through the formation of a ternary complex consisting of the enzyme, the substrate, and timolol. These results indicate that timolol binds apart from the narrow cavity of the active site. AutoDocking results showed that timolol binds at the entrance of the active site cavity in a region where the proton shuttle residue, His 64, of HCA I or II, is placed. The enzyme kinetic and AutoDocking results showed that timolol might weakly bind near the proton shuttle residue, His 64, to accelerate the proton transfer rate from His 64 to the buffer components. It is known that efficient activators of carbonic anhydrase possess a bulky aromatic/heterocyclic moiety and a primary/secondary amino group in their molecular structure. Timolol has a heterocyclic moiety and a secondary amino group, which are typical structures in efficient activators of carbonic anhydrase.

Key words timolol; carbonic anhydrase; activator; enzyme kinetics; AutoDock simulation

Therapy for glaucoma is generally individualized for each glaucoma patient, with several factors, including intraocular pressure (IOP) and the degree of optic nerve damage, given consideration. In the treatment of glaucoma, several types of agents such as beta-blockers, prostaglandin analogues and carbonic anhydrase (CA) inhibitors are clinically used at present in pharmacotherapy. ${ }^{1-2)}$ Beta-blockers or prostaglandin F2-alpha analogues have been shown to be effective ocular hypotensive agents when used alone in ocular hypertensive or open-angle glaucoma patients. ${ }^{3,4)}$ These two types of agents are currently recognized as the first-line medications for treating glaucoma. ${ }^{5)}$ However, mono-therapy with timolol, a beta-blocker, or latanoprost, a prostaglandin analogue, is often insufficient to maintain a desirable IOP over the long term in many patients. ${ }^{6}$ In these cases, CA inhibitors are often used as an adjunctive therapy to timolol or to latanoprost in order to achieve a desirable IOP. ${ }^{7)}$ Although it has been shown that monotherapy with timolol results in a smaller IOP reduction than monotherapy with latanoprost, the combination therapies of either timolol or latanoprost with CA inhibitors show comparable IOP reduction activity in glaucoma patients. ${ }^{8)}$ These clinical observations suggest that there exist different interactions between CA inhibitors and latanoprost or timolol that affect IOP reduction.

In our previous paper, ${ }^{9)}$ latanoprost was shown in an in vitro study to be a weak noncompetitive inhibitor of human carbonic anhydrase (HCA) I and II and to bind to the zinc ion, which plays an important role in the expression of enzyme activity according to the AutoDocking simulation method.

In the present study, to elucidate the interaction mechanism between timolol and $\mathrm{CA}$ inhibitors, the reaction mecha- nism and binding mode of timolol with respect to CA were investigated by enzyme-kinetic studies and an AutoDocking simulation method.

\section{MATERIALS AND METHODS}

Materials Human CA I and II (HCA I and HCA II) were purchased from Sigma Co., Ltd. Since the purities of HCA I and II were shown to be more than $95 \%$ by sodium dodecyl sulfate-polyacrylamide gel electrophoresis (SDSPAGE), these enzymes were used without further purification. Timolol hemihydrate (formal name: $(2 S)-1-[(1,1-d i-$ methylethyl)amino]-3-[\{4-(4-morpholinyl)-1,2,5-thiadiazol3-yl \}oxy]-2-propanol 1/2 $\mathrm{H}_{2} \mathrm{O}$ ) was provided by Santen Pharmaceutical Co. Ltd. All other reagents used in this experiment were of analytical grade and were purchased from Wako Pure Chemical Co., Ltd. (Tokyo, Japan).

Determination of the Concentration of $\mathbf{C O}_{2} \quad \mathrm{The}^{\mathrm{CO}_{2}}$ concentration of pure water saturated with $\mathrm{CO}_{2}$ was measured using a $\mathrm{CO}_{2}$ electrode (Orion 9502BN) connected to an Orion Ion-meter $720 \mathrm{~A}$. The standard solutions of $\mathrm{CO}_{2}$ were prepared using $0.5-5 \mathrm{ml}$ of $0.1 \mathrm{M} \mathrm{NaHCO}_{3}$ solutions mixed with $5 \mathrm{ml}$ of $1 \mathrm{M}$ citric acid buffer ( $\mathrm{pH} 4.5$ ) and filled up to $50 \mathrm{ml}$ with pure water.

Determination of the Enzyme Activity of Carbonic Anhydrase Determination of the enzyme activity was based on the hydration of $\mathrm{CO}_{2}$ catalyzed by HCA I and II. The technique used for this purpose was the colorimetric technique of changing $\mathrm{pH}$ with the stopped flow method. ${ }^{10)}$ Phenol red $\left(3 \times 10^{-5} \mathrm{M}\right)$ was used as a $\mathrm{pH}$ indicator, and 0.01 M $N$-(2-hydroxyethyl)piperazine- $N^{\prime}$-2-ethanesulfonic acid (HEPES) was used as a buffer at $\mathrm{pH} 7.4,25^{\circ} \mathrm{C}$. Ionic 
strength $(I=0.1)$ was maintained by using $\mathrm{Na}_{2} \mathrm{SO}_{4}$. Kinetic studies of the hydration of $\mathrm{CO}_{2}$ catalyzed by HCA were monitored at $435 \mathrm{~nm}$ by a micro-stopped flow apparatus (WakenOtsuka Electronics) connected to a microcomputer. At least five kinetic traces were averaged for each experiment at a given reagent concentration. Kinetic data were analyzed and processed by KaleidoGraph (Hulinks, Tokyo, Japan). The hydration velocity of $\mathrm{CO}_{2}$ was obtained using the initial rate of the reaction curve traced by stopped flow. $\mathrm{HCO}_{3}^{-}$concentrations produced by the enzyme reaction were calculated from the absorbance changes of phenol red at $435 \mathrm{~nm}$ using the following equation obtained by the method of Khalifah R. G. ${ }^{11)}$ :

$\left[\mathrm{HCO}_{3}^{-}\right]=0.184 \times \Delta A$

$\Delta A$ : the absorbance change of phenol red at $435 \mathrm{~nm}$

Docking Simulation of the Complex of Timolol with HCA I or II The AutoDock 3.0.5 docking program ${ }^{12)}$ was used for the complex of timolol with HCA I or II. The crystal structures of HCA I (1HUH, Resolution $2.2 \AA^{13)}$ ) and HCA II (1RAZ, Resolution $1.9 \AA^{14)}$ ) were obtained from the Protein Data Bank. The polar hydrogen, partial charge, and solvation parameters for use with the AutoDock 3.0.5 simulation were applied using AutoDock Tools. ${ }^{15)}$ The geometry of timolol as a ligand molecule was optimized by the semiempirical MNDO-AM1 ${ }^{16)}$ method. For further docking simulations, the Gasteiger atomic charges calculated by the AutoDock Tools program were utilized as the partial atomic charges of the ligand molecule. The docking simulations were carried out with rigid HCA I and II and a flexible ligand. The most suitable structure for the flexible ligand molecule was then optimized by the rotation of all single bonds in the ligand molecule. The grid parameter file and the docking parameter file were set up using the AutoDockTools program. A grid of $127 \times 127 \times 127$ points in the $x$-, $y$-, and $z$-axis directions was built, and the grid center was at the center of HCA I and II. The maximum number of energy evaluations was repeated up to 30 million times in the present simulations. The positional root mean square deviation tolerance (rmstol) used in a conformational cluster analysis was set to cut off at $0.5 \AA$. Default settings were used for all other parameters.

Docking simulations were carried out by AutoDock using a Lamarckian genetic algorithm. After the docking simulation came to an end, a cluster analysis was carried out with regard to all conformations calculated by the docking simulation. The root mean square deviation (rmsd) value using the cluster analysis was calculated using the rmsd of the interatomic distance between the cognate atoms of two conformations. If the rmsd of two conformations was within the rmstol $(0.5 \AA)$, these conformations were classified into the same cluster. Finally, the HCA-timolol complex with the most stable energy was adopted as the most favorable structure of the complex for HCA I and II. All calculations in the present study were carried out on the NEC PC-Parallel Cluster System at the Green Science Research Center of Fukuyama University and on an HIT HPC-IAX at our laboratory.

\section{RESULTS}

The Influence of Timolol on the Enzyme Activities of HCA I and HCA II The influence of timolol on the en-

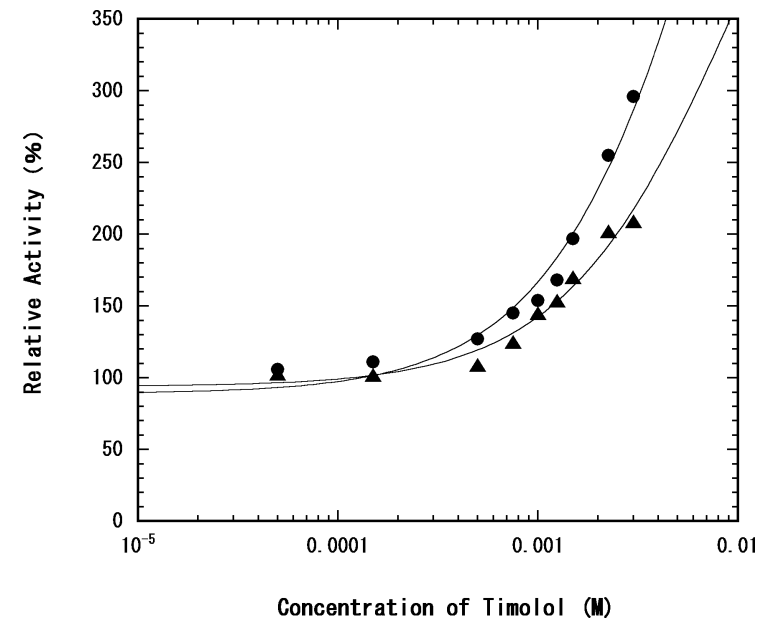

Fig. 1. The Activation of HCA I and II Hydration Activities in the Presence of Various Concentrations of Timolol

The relative activity of HCA I or HCA II in the presence of timolol compared to that in the absence of timolol ( $v$ in the presence of timolol $) /(v$ in the absence of timolol $) \times 100 \%)$ is plotted against the concentrations of timolol. - - , HCA I; - $\mathbf{-}-$, HCA II.

zyme activities (the initial velocity, $v$ ) of HCA I and II was determined at $25^{\circ} \mathrm{C}$ in $0.01 \mathrm{M}$ HEPES buffer ( $\mathrm{pH}$ 7.4: $I=0.1$ : phenolred $=3 \times 10^{-5} \mathrm{M}$ ). The enzyme activities of HCA I and HCA II were measured at a constant substrate concentration $\left(\mathrm{CO}_{2}: 2.25 \times 10^{-2} \mathrm{M}\right)$ in the presence of various concentrations of timolol $\left(0-3 \times 10^{-3} \mathrm{M}\right)$. The relative activities of HCA I and HCA II in the presence of timolol compared to those in the absence of timolol are plotted as the concentrations of timolol (Fig. 1).

In Fig. 1, the relative activities of HCA I and II increased as the concentrations of timolol increased. This behavior shows that timolol activates the enzyme activities of HCA I and HCA II.

Type of Activation of Timolol for HCA I The initial velocity, $v$, at a substrate concentration, $s$, was measured for five different values of $s$ in the absence and presence of timolol $\left(0 \mathrm{M}, 1.5 \times 10^{-3} \mathrm{M}, 3 \times 10^{-3} \mathrm{M}\right)$. The relationships between the initial velocity $(v)$ and the substrate concentration $(s)$ in the absence and presence of various concentrations of timolol are shown in Fig. 2A and give hyperbolic functions.

To obtain $K_{\mathrm{m}}$ and $V_{\max }$, the Michelis-Menten equation $\left(v=\left(V_{\max \cdot s}\right) /\left(s+K_{\mathrm{m}}\right)\right)$ was fitted to the relationships between $s$ and $v$ by the nonlinear least square method using the program KaleidaGraph. The values of HCA I in the absence of timolol were as follows: $K_{\mathrm{m}}=0.019 \pm 0.007 \mathrm{M}, \quad V_{\max }=0.019 \pm$ $0.004 \mathrm{M} \mathrm{s}^{-1}$, and $k_{0}=1.4( \pm 3) \times 10^{5} \mathrm{~s}^{-1}$. These values are very similar to those obtained in the previous paper. ${ }^{8)}$ The $K_{\mathrm{m}}$ values in the presence of $1.5 \times 10^{-3} \mathrm{M}$ and $3 \times 10^{-3} \mathrm{M}$ timolol were $0.020 \pm 0.002$ and $0.020 \pm 0.005 \mathrm{M}$, respectively. The values of $K_{\mathrm{m}}$ are almost the same in the absence of timolol as they are in the presence of timolol. The $V_{\max }$ values in the presence of $1.5 \times 10^{-3} \mathrm{M}$ and $3 \times 10^{-3} \mathrm{M}$ timolol were $0.029 \pm 0.001$ and $0.037 \pm 0.006 \mathrm{M} \mathrm{s}^{-1}$, respectively. The values of $V_{\max }$ increased as the concentration of timolol was increased.

The type of activation that timolol exerted on HCA I at $25^{\circ} \mathrm{C}$, pH 7.4 (0.01 м HEPES buffer: $\left.I=0.1\right)$ was determined by Lineweaver-Burks $(1 / s-1 / v), s / v-s$, and $v-v / s$ plots, which are shown by Eqs. 1, 2 and 3 below, respectively. ${ }^{17,18)}$ 

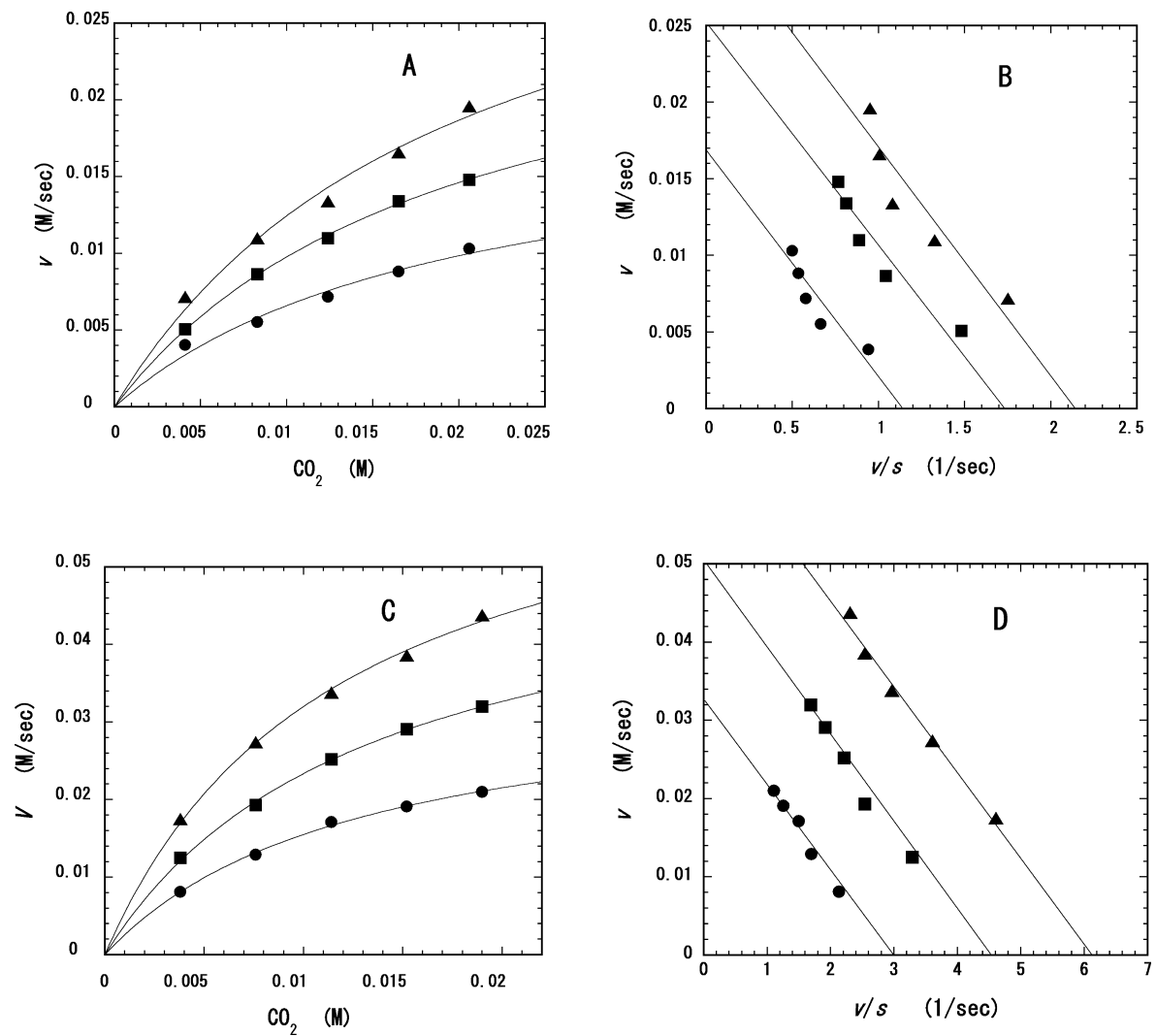

Fig. 2. $\quad v-s$ and $v-v / s$ Plots in HCA I and HCA II

(A) Relationship between $v$ and $s$ in HCA I. The enzyme reaction was monitored in $0.01 \mathrm{M}$ HEPES buffer $\left(\mathrm{pH} 7.4, I=0.1\left(\mathrm{Na}_{2} \mathrm{SO}_{4}\right)\right)$ containing phenol red $\left(3 \times 10^{-5} \mathrm{M}\right)$ at $25^{\circ} \mathrm{C}$. The concentrations of timolol hemihydrate were as follows: - - $0 \mathrm{M} ;-\square-1.5 \times 10^{-3} \mathrm{M} ;-\boldsymbol{\Lambda}-, 3.0 \times 10^{-3} \mathrm{M}$. The values of $K_{\mathrm{A}}(0.012 \mathrm{M}), \alpha(5.7), K_{\mathrm{m}}(0.019 \mathrm{M}), V_{\max }$ $\left(0.019 \mathrm{M} \mathrm{s}^{-1}\right)$ and the concentration of timolol $\left.\left(0 \mathrm{M}, 1.5 \times 10^{-3} \mathrm{M}, 3.0 \times 10^{-3} \mathrm{M}\right)\right)$ were inserted into Eq. 4 to obtain the theoretical curves between $v$ and $s$. The theoretical curves are shown as the solid lines. (B) $v-v / s$ plots in HCA I. The concentrations of timolol hemihydrate were as follows: -,$- 0 \mathrm{M} ;-\square-, 1.5 \times 10^{-3} \mathrm{M} ;-\mathbf{\Lambda}-, 3.0 \times 10^{-3} \mathrm{M}$. (C) Relationship between $v$ and $s$ in HCA II. The enzyme reaction was monitored in $0.01 \mathrm{M}$ HEPES buffer $\left(\mathrm{pH} 7,4, I=0.1\left(\mathrm{Na}_{2} \mathrm{SO}_{4}\right)\right)$ containing phenol red $\left(3 \times 10^{-5} \mathrm{M}\right)$ at $25^{\circ} \mathrm{C}$. The concentrations of timolol hemihydrate were as follows: - $-0 \mathrm{M} ;-\square-2 \times 10^{-3} \mathrm{M} ;-\boldsymbol{-}-, 4.5 \times 10^{-3} \mathrm{M}$. The values of $K_{\mathrm{A}}(0.0093 \mathrm{M}), \alpha(4.0), K_{\mathrm{m}}(0.013 \mathrm{M}), V_{\max }\left(0.035 \mathrm{M} \mathrm{s}^{-1}\right)$ and the concentration of timolol $\left.\left(0 \mathrm{M}, 2 \times 10^{-3} \mathrm{M}, 4.5 \times 10^{-3} \mathrm{M}\right)\right)$ were inserted into Eq. 4 to obtain the theoretical curves between $v$ and $s$. The theoretical curves are shown as the solid lines. (D) $v-v / s$ plots in HCA II. The concentrations of timolol hemihydrate were as follows: $-\square, 0 \mathrm{M} ;-\square-2 \times 10^{-3} \mathrm{M} ;-\mathbf{\square}-, 4.5 \times 10^{-3} \mathrm{M}$.

$$
\begin{aligned}
& 1 / v=\left(K_{\mathrm{m}} / V_{\max }\right)(1 / s)+1 / V_{\max } \\
& s / v=\left(1 / V_{\max }\right) s+\left(K_{\mathrm{m}} / V_{\max }\right) \\
& v=-K_{\mathrm{m}}(v / s)+V_{\text {max }}
\end{aligned}
$$

Lineweaver-Burks $(1 / s-1 / v), s / v-s$, and $v-v / s$ plots in the absence and presence of timolol were used to determine the activation mechanism of timolol. In the Lineweaver-Burks $(1 / v-1 / s)$ plot, all lines were crossed at a point on the $1 / s$ axis (data not shown). In the $s / v-s$ plot, all lines were also crossed at a point on the $s$ axis (data not shown). In the $v-v / s$ plot, all lines were parallel lines with the same slopes (Fig. 2B). These behaviors clearly indicate that timolol only changes $V_{\max }$ and does not change the value of $K_{\mathrm{m}}$ of $\mathrm{CO}_{2}$ for HCA I. In HCA I, timolol noncompetitively activates the $\mathrm{CO}_{2}$ hydration activity of HCA I.

Kinetic results clearly show that timolol increases the value of $V_{\max }$ but does not influence $K_{\mathrm{m}}$. On the basis of these results, the activation mechanism of timolol on HCA is proposed as shown in Chart 1 in which E, S, P, and A are the enzyme, the substrate, the product and the activator, respectively, and the rate constants of $k_{+2}$ and $\alpha k_{+2}$ for two forms of the enzyme-substrate adducts of ESA and ES are different.

In Chart $1, \alpha$ is larger than 1.0 because $\mathrm{A}$ is an activator and the rate constant $\left(\alpha k_{+2}\right)$ for ESA is larger than that $\left(k_{+2}\right)$

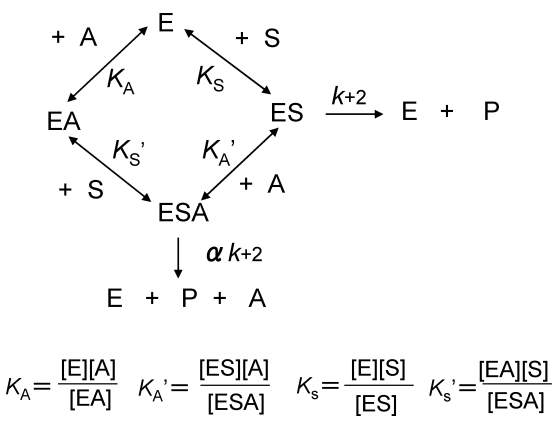

Chart 1. The Activation Mechanism of Timolol for HCA Hydration Activity

E, S, P, and A are the enzyme, the substrate, the product and the activator, respectively.

of ES. If rapid equilibrium is set up among the four species (E, ES, EA, and ESA), $K_{\mathrm{S}}^{\prime} K_{\mathrm{A}}$ should be equal to $K_{\mathrm{S}} K_{\mathrm{A}}^{\prime}$. If $K_{\mathrm{A}}$ is equal to $K_{\mathrm{A}}^{\prime},{ }^{17,18)}$ the following equation is obtained:

$$
v=\frac{V_{\max } \frac{\left(1+\alpha[\mathrm{A}] / K_{\mathrm{A}}\right)}{\left(1+[\mathrm{A}] / K_{\mathrm{A}}\right)} s}{s+K_{\mathrm{m}}}
$$

Equation 4 clearly indicates that the value of $K_{\mathrm{m}}$ does not change, but the values of $V_{\max }$ change with the addition of the 
activator. Therefore, the relationships between $v$ and $s$ or $v$ and $s / v$ in Figs. 2A and B are completely explained by Eq. 4. Equation 4 clearly shows that timolol does not influence the value of $K_{\mathrm{m}}$ and increases the value of $V_{\max }$. These results indicate that timolol is the activator of HCA I and that $\alpha$ in Chart 1 is larger than 1.0. This implies that the rate $\left(\alpha k_{+2}\right.$ in Chart 1$)$ at which the ternary complex (ESA in Chart 1) produces the product $(P)$ is faster than that $\left(k_{+2}\right)$ of the enzymesubstrate complex (ES in Chart 1). To obtain the values of $\alpha$ and the dissociation constants $\left(K_{\mathrm{A}}\right)$ of timolol, $V_{\max }\left(1+\alpha[\mathrm{A}] / K_{\mathrm{A}}\right) /\left(1+[\mathrm{A}] / K_{\mathrm{A}}\right)$ in Eq. 4 was fitted to the relationships between the concentration of timolol and $V_{\max }$ by the nonlinear least square method using the program KaleidaGraph. The values of the dissociation constant $\left(K_{\mathrm{A}}\right)$ and $\alpha$ of timolol were $0.012 \mathrm{M}$ and 5.7 for HCA I, respectively. The values of $K_{\mathrm{A}}(0.012 \mathrm{M}), \alpha(5.7), K_{\mathrm{m}}(0.019 \mathrm{M}), V_{\max }$ $\left(0.019 \mathrm{M} \mathrm{s}^{-1}\right)$ and the concentrations of timolol $(0 \mathrm{M}$, $1.5 \times 10^{-3} \mathrm{M}, 3.0 \times 10^{-3} \mathrm{M}$ ) were inserted into Eq. 4 to obtain the theoretical curves between $v$ and $s$. The theoretical curves are shown in Fig. 2A. In Fig. 2A, the theoretical curves are very consistent with the data obtained by the enzyme kinetics, respectively.

Timolol does not influence the $K_{\mathrm{m}}$ value and only increases the $V_{\max }$ value. Therefore, timolol should bind apart from the substrate binding site, and the binding of timolol to the enzyme does not disturb the binding of the substrate, but timolol accelerates the $\mathrm{CO}_{2}$ hydration activity of HCA I.

Type and Mechanism of Activation of Timolol for HCA II In HCA II, the initial velocity, $v$ at a substrate concentration, $s$, was also measured for five different values of $s$ in the absence and presence of timolol $\left(0 \mathrm{M}, 2.0 \times 10^{-3} \mathrm{M}, 4.5 \times\right.$ $\left.10^{-3} \mathrm{M}\right)$. The relationships between $v$ and $s$ in the absence and presence of various concentrations of timolol are shown in Fig. 2C and give hyperbolic functions. To obtain $K_{\mathrm{m}}$ and $V_{\max }$, the Michelis-Menten equation $\left(v=\left(V_{\max \cdot s}\right) /\left(s+K_{\mathrm{m}}\right)\right)$ was also fitted to the relationships between $s$ and $v$ by the nonlinear least square method using the program KaleidaGraph. The values for HCA II in the absence of timolol were as follows: $K_{\mathrm{m}}=0.013 \pm 0.001 \mathrm{M}, V_{\max }=0.035 \pm 0.002 \mathrm{M} \mathrm{s}^{-1}$, and $k_{0}=1.4( \pm 0.1) \times 10^{5} \mathrm{~s}^{-1}$. These values are very similar to those obtained in the previous paper. ${ }^{9)}$ The $K_{\mathrm{m}}$ values in the presence of $2.0 \times 10^{-3} \mathrm{M}$ and $4.5 \times 10^{-3} \mathrm{M}$ timolol were $0.013 \pm 0.001$ and $0.012 \pm 0.001 \mathrm{M}$, respectively. The values of $K_{\mathrm{m}}$ are almost the same in the absence of timolol as they are in the presence of timolol. The $V_{\max }$ values in the presence of $2.0 \times 10^{-3} \mathrm{M}$ and $4.5 \times 10^{-3} \mathrm{M}$ timolol were $0.054 \pm 0.002 \mathrm{M} \mathrm{s}^{-1}$ and $0.070 \pm 0.003 \mathrm{M} \mathrm{s}^{-1}$, respectively. The values of $V_{\max }$ increased as the concentration of timolol was increased. These behaviors are completely identical to those of HCA I.

The type of activation that timolol exerted on HCA II was also determined by Lineweaver-Burks $(1 / s-1 / v), s / v-s$, and $v-v / s$ plots, which are shown by Eqs. 1, 2 and 3, respectively. ${ }^{17)}$ In the $v-v / s$ plot, all lines were parallel lines with the same slopes (Fig. 2D). The activation mechanism of timolol for HCA II is completely consistent with that for HCA I. Therefore, timolol activates HCA II on the basis of the reaction mechanism shown in Chart 1.

The values of the dissociation constant $\left(K_{\mathrm{A}}\right)$ and $\alpha$ of timolol for HCA II were also calculated from the relationships between the concentration of timolol and $V_{\max }$ and were $0.0093 \mathrm{M}$ and 4.0 , respectively. The $\alpha$ value of HCA II is smaller than that of HCA I. The values of $K_{\mathrm{A}}(0.0093 \mathrm{M}), \alpha$ (4.0), $K_{\mathrm{m}}(0.013 \mathrm{M}), V_{\max }\left(0.035 \mathrm{M} \mathrm{s}^{-1}\right)$ and the concentrations of timolol $\left.\left(0 \mathrm{M}, 2 \times 10^{-3} \mathrm{M}, 4.5 \times 10^{-3} \mathrm{M}\right)\right)$ were inserted into Eq. 4 to obtain the theoretical curves between $v$ and $s$. The theoretical curves are shown in Fig. 2C. In Fig. 2C, the theoretical curves are very consistent with the data obtained by the enzyme kinetics, respectively.

AutoDock Simulation of the Timolol-HCA I or II Complex AutoDock simulation of the timolol-HCA I or II complex was performed to determine why and how timolol activates the enzyme activity of CA. The most favorable interaction modes of the HCA I-timolol and HCA II-timolol complexes calculated by the AutoDock simulation are shown in Fig. 3.

In Fig. 3A, the structure of the timolol-HCA I complex was superimposed onto that of the timolol-HCA II complex, because the identity of the amino acid sequences between HCA I and II is $60 \%$ on the basis of the result obtained from the FASATA www system. The rounded ribbon model structures of HCA I and II are shown in white and magenta, respectively, and the zinc ions located at the active sites are
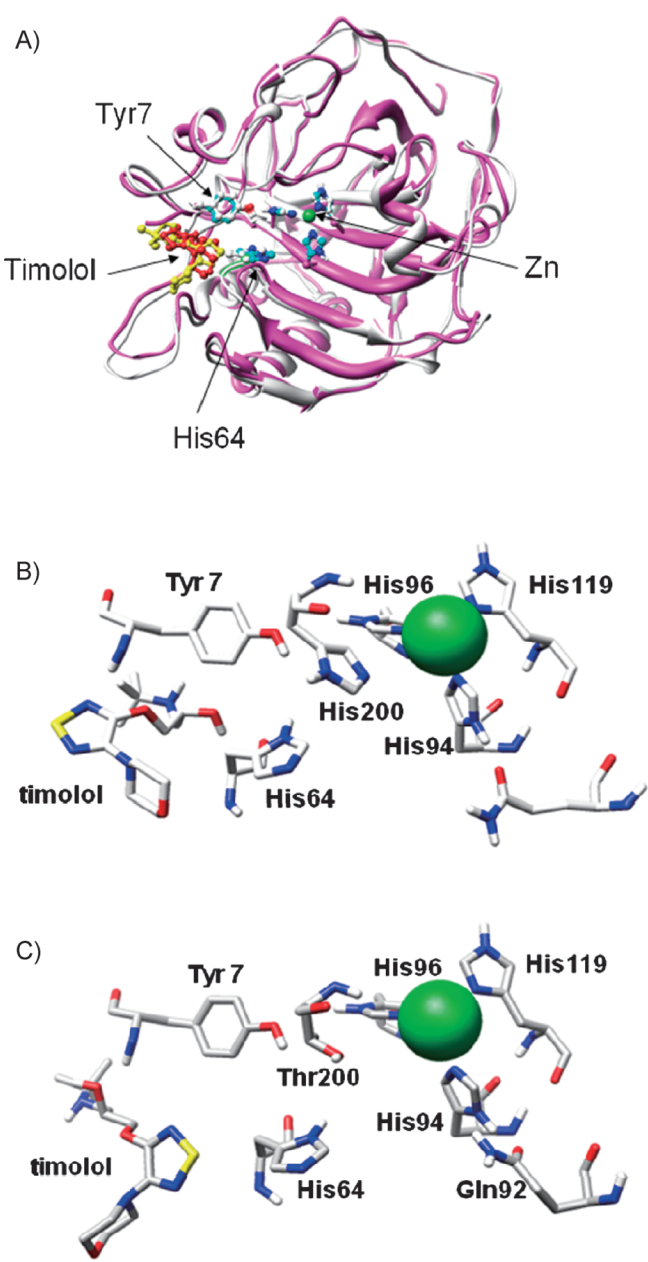

Fig. 3. The Structure of the Timolol-HCA I or II Complex

(A) The structure of the timolol-HCA I complex superimposed onto that of the timolol-HCA II complex. The rounded ribbon model structures of HCA I and II are shown in white and magenta, respectively, and the zinc ions located at the active sites are shown as a green space fill model. Timolols which bind to HCA I and II are shown as respective red and yellow ball-and-stick models. The important residues, which are Tyr 7 and His 64, 94, 96, and 119, are shown as ball-and-stick models. Timolols are bound near His-64 residues, which are the proton shuttle residues in HCA I and II. (B) Active site residues in HCA I with timolol. (C) Active site residues in HCA II with timolol. 
shown as a green space fill model. Timolols which bind to HCA I and II are shown respectively as red and yellow balland-stick models. In Fig. 3A, the important residues, which are Tyr 7 and His 64, 94, 96, and 119, are shown as ball-andstick models, and timolols are bound near His-64 residues in HCA I and II. Timolols are bound far from the substrate binding sites around the zinc ions and at the entrances of the active site cavities in the region where the proton shuttle residue, His 64 , is placed.

Timolols and the important residues in the active site of HCA I and II are shown in Figs. 3B and 3C. The arrangement of the residues in HCA I was very similar to that in HCA II except that His 200 of HCA I was replaced by Thr 200 in HCA II. The binding mode of timolol to HCA I was slightly different from that to HCA II.

\section{DISCUSSION}

Supuran ${ }^{19)}$ recently reported many inhibitors and activators of CA. Many X-ray crystallographic structures of the inhibitor and activator adducts of HCA I and II also have been reported and have shown that the activators of $\mathrm{CA}$ bind at the entrance of the active site cavity in the region where the proton shuttle residue His 64 is placed, whereas the inhibitors of CA (sulphoamide derivatives, etc.) bind to the catalytic zinc ions displacing the fourth ligand, a hydroxide ion/water molecule acting as a nucleophile in catalysis. ${ }^{20-24)}$ It is known that efficient activators of CA possess a bulky aromatic/heterocyclic moiety and a primary/secondary amino group in their molecular structure. ${ }^{10)}$

Timolol, a beta-blocker, activates the enzyme activity of HCA I or II. The enzyme kinetic method showed that timolol activates HCA I and II activities through the formation of a ternary complex (ESA in Chart 1) consisting of the enzyme, the substrate, and timolol. The enzyme kinetic results showed that the binding of timolol does not influence the affinity of the substrate binding to the enzymes. These results indicate that timolol binds apart from the substrate binding cavity.

AutoDocking results showed that timolol binds at the entrance of the active site cavity in the region where the proton shuttle residue, His 64 , is placed. The binding site of timolol is far from the substrate binding site around the zinc ion. This behavior is completely consistent with the results presumed by the enzyme kinetic method.

In $\mathrm{CA}$, the rate-determining step in catalysis is the proton transfer reactions between the active site and the reaction medium. ${ }^{10,20)}$ Timolol, which possess a bulky aromatic/heterocyclic moiety and a secondary amino group in its molecular structure $\left(\mathrm{p} K_{\mathrm{a}}=9.2^{25)}\right)$, is bound to the entrance of the active site of CA where it may actively participate in shuttling protons between the active site and the bulk solvent. ${ }^{10,20)}$

Because of the weaker IOP reduction, CA inhibitor is frequently used as an adjunctive agent to other anti-glaucoma agents such as timolol or latanoprost. Ophthalmologists suggested that monotherapy patients treated with timolol generally show a smaller IOP reduction than those treated with latanoprost. ${ }^{4)}$ In spite of the fact that timolol shows weaker IOP reduction activity than latanoprost in monotherapy treatment, the combination treatment with timolol and a CA inhibitor showed IOP reduction activity comparable to that of the combination treatment with latanoprost and a CA inhibitor. ${ }^{8)}$
In our previous paper, ${ }^{9)}$ latanoprost was observed to act as a weak inhibitor of CA. On the other hand, the present study has shown that timolol acts as an activator of CA. The inhibition constants of latanoprost $\left(K_{\mathrm{i}}=0.24 \mathrm{~mm}\right.$ for HCA I and $2.3 \mathrm{~mm}$ for HCA II $)^{9)}$ and the activation constants of timolol $\left(K_{\mathrm{A}}=12 \mathrm{~mm}\right.$ for HCA I and $9.3 \mathrm{~mm}$ for HCA II) are much larger than the inhibition constants of brinzolamide $\left(K_{\mathrm{i}}=\right.$ $32 \mathrm{nM}$ for HCA I and $K_{\mathrm{i}}=0.13 \mathrm{~nm}$ for HCA II), which is an HCA inhibitor used as an ocular hypotensive agent. These findings might suggest that these drugs have comparable IOP reduction activity when combined with CA inhibitors. However, the small affinity of timolol or latanoprost for CA does not seem to affect CA activity in such a way as to have substantial therapeutic effects. Therefore, the comparable IOP reduction activities achieved by combination treatments, in spite of their different basal IOP reduction activities, cannot be explained by the direct effects of timolol or latanoprost on CA activity. Previously, we have shown that latanoprost binds to the zinc ion that is located in the center cavity of the CA enzyme and plays an important role in the expression of the enzyme activity. ${ }^{9)}$ On the other hand, the binding site of timolol has been shown in the present study to be far from the substrate binding site around the zinc ion. If the binding modes of timolol or latanoprost cause structural and/or functional change in $\mathrm{CA}$, this might explain the different drugdrug interactions of these drugs with CA inhibitors.

It is very interesting to investigate how the binding mode affects the drug-drug interaction between CA inhibitors and timolol or latanoprost. Further studies of CA inhibitor activity under comparable conditions of timolol or latanoprost that binds to a different site of CA should be performed.

Acknowledgements This research was partially supported by the High-Tech Research Center Project for Private Univ., and a matching fund subsidy from the Ministry of Education, Culture, Sports, Science and Technology of Japan, 2004-2008.

\section{REFERENCES}

1) Whitson J. T., Expert Opin. Pharmacother, 8, 3237-3249 (2007).

2) Khouri A. S., Realini T., Fechtner R. D., Drugs Aging, 24, 10071016 (2007).

3) Bannon J. A., Stewart K. A., DeLisser O., Schrogie J. J., Arch. Intern. Med., 146, 654-657 (1986).

4) Bucci M. G., Italian Latanoprost Study Group., J. Glaucoma, 8, 24 30 (1999)

5) Watson P., Stjernschantz J., Ophthalmology, 103, 126-137 (1996).

6) Adamsons I., Clineschmidt C., Polis A., Taylor J., Shedden A., Laibovitz R., J. Glaucoma, 7, 253-260 (1998).

7) Tsukamoto H., Noma H., Matsuyama S., Ikeda H., Mishima H. K., J. Ocul. Pharmacol. Ther., 21, 170-173 (2005).

8) Miura K., Ito K., Okawa C., Sugimoto K., Matsunaga K., Uji Y., J. Glaucoma, 17, 233-237 (2008).

9) Sugimoto A., Ikeda H., Tsukamoto H., Kihara K., Takeda C., Hirose J., Hata T., Ono Y., Biol. Pharm. Bull., 31, 796-801 (2008).

10) Vullo D., Innocenti A., Nishimori I., Scozzafava A., Kaila K., Supuran C.T., Bioorg. Med. Chem. Lett., 17, 4107-4112 (2007).

11) Khalifah R. G., J. Biol. Chem., 246, 2561-2573 (1971).

12) Morris G. M., Goodsell D. S., Halliday R. S., Huey R., Hart W. E., Belew R. K., Olson A. J., J. Comput. Chem., 19, 1639-1662 (1998).

13) Kumar V., Kannan K. K., Sathyamurthi P., Acta Crystallogr. D: Biol. Crystallogr., D50, 731—738 (1994).

14) Jöensson B. M., Håkansson K., Liljas A., FEBS Lett., 322, 186-190 (1993). 
15) Sanner M. F., J. Mol. Graphics Mod., 17, 57—61 (1999).

16) Dewar M. J. S., Zoebisch E. G., Healy E. F., Stewart J. J. P., J. Am. Chem. Soc., 107, 3902-3909 (1985)

17) Cornish-Bowden A., "Fundamentals of Enzyme Kinetics," Butterworth \& Co., London, 1981.

18) Hiromi K. "Introduction to Enzyme Chemistry," ed. by Nishizawa K., Shimura K., Nankodo, Tokyo, 1995, pp. 21-92.

19) Supuran C. T., Nature (London), 7, 168-181 (2008).

20) Briganti F., Mangani S., Orioli P., Scozzafava A., Vernaglione G., Supuran C. T., Biochemistry, 36, 10384-10392 (1997).
21) Scozzafava A., Supuran C. T., Bioorg. Med. Chem. Lett., 12, 11771180 (2002).

22) Temperini C., Scozzafava A., Puccetti L., Supuran C. T., Bioorg. Med. Chem. Lett., 15, 5136-5141 (2005).

23) Temperini C., Scozzafava A., Supuran C. T., Bioorg. Med. Chem. Lett., 16, 5152-5156 (2006).

24) Temperini C., Innocenti A., Scozzafava A., Mastrolorenzo A., Supuran C. T., Bioorg. Med. Chem. Lett., 17, 628-635 (2007).

25) Tarvainen T., Malin M., Barragan I., Tuominen J., Seppaelae J., Jaervinen K., Int. J. Pharm., 310, 162-167 (2006). 